\title{
PAPER
}

\section{Detection of grey matter loss in mild Alzheimer's disease with voxel based morphometry}

\author{
G B Frisoni, C Testa, A Zorzan, F Sabattoli, A Beltramello, H Soininen, M P Laakso
}

J Neurol Neurosurg Psychiatry 2002;73:657-664

See end of article for authors' affiliations

Correspondence to:

$\operatorname{Dr} G$ B Frisoni, Laboratory

of Epidemiology and

Neuroimaging, IRCCS San

Giovanni di Dio-FBF, via

Pilastroni 4, 25125

Brescia, Italy;

papers@centroAlzheimer.it

Received

19 February 2002

In revised form 14 June

2002

Accepted 8 August 2002

\begin{abstract}
Objectives: To test the applicability of an automated method of magnetic resonance image analysis (voxel based morphometry) to detect presence and severity of regional grey matter density reduction - a proxy of atrophy-in Alzheimer's disease.

Methods: Twenty nine probable Alzheimer's patients and 26 non-demented controls (mini-mental state examinations mean (SD) 21 (4) and 29 (1)) underwent high resolution 3D brain magnetic resonance imaging. Spatial normalisation to a stereotactic template, segmentation into grey matter, white matter, and cerebrospinal fluid, and smoothing of the grey matter were carried out based on statistical parametric mapping (SPM99) algorithms. Analyses were carried out: (a) contrasting all Alzheimer's patients with all controls ( $p<0.05$ corrected for multiple comparisons); (b) contrasting the three Alzheimer's patients with mini-mental state of 26 and higher with all controls $(p<0.0001$ uncorrected); and (c) correlating grey matter density with mini-mental state score within the Alzheimer's group $(p<0.0001$ uncorrected).

Results: When all Alzheimer's patients were compared with controls, the largest atrophic regions corresponded to the right and left hippocampal/amygdalar complex. All parts of the hippocampus (head, body, and tail) were affected. More localised atrophic regions were in the temporal and cingulate gyri, precuneus, insular cortex, caudate nucleus, and frontal cortex. When the mildest Alzheimer's patients were contrasted with controls, the hippocampal/amygdalar complex were again found significantly atrophic bilaterally. The mini-mental state score correlated with grey matter density reduction in the temporal and posterior cingulate gyri, and precuneus, mainly to the right.

Conclusions: Voxel based morphometry with statistical parametric mapping is sensitive to regional grey matter density reduction in mild Alzheimer's disease.
\end{abstract}

O $\mathrm{f}$ the biological indicators of Alzheimer's disease (AD), those detecting medial temporal lobe atrophy based on three dimensional Tl weighted magnetic resonance (MR) imaging are among the most sensitive. Hippocampal and entorhinal cortex volumetry and surface measures of the entorhinal cortex are believed to have high sensitivity and specificity in the detection of AD from non-demented elderly controls $^{12}$ and can predict conversion of mild cognitive impairment to $\mathrm{AD}^{3}$ However, the variability of the measurements because of the human tracer has so far limited direct comparisons of the results of different research groups. The development of observer independent tools might be a significant advancement.

To date, some computer based tools are available that can detect volume or shape changes in the regions of interest to $\mathrm{AD}^{4-7}$ Among these, one of the most user independent is statistical parametric mapping (SPM). ${ }^{8}$ Initially developed for brain activation studies with positron emission tomography, ${ }^{9}$ it has been applied to different image analysis issues. In the case of morphometry, SPM permits the comparison of the density of grey matter on a voxel by voxel basis in a group of patients with that of a group of controls. The result of the comparison is a three dimensional map of significant regional differences of grey matter density reduction, a close proxy of grey matter atrophy. The process is largely automatic and human intervention is comparatively low. Three studies have been published so far on $\mathrm{AD}^{10-12}$ but inconsistent results, different study populations and analysis protocols, and the present availability of more sophisticated image processing protocols ${ }^{13}$ argue for an independent confirmation.

The aim of this study is to test the sensitivity of voxel based morphometry with SPM in the detection of grey matter density reduction and its association with global disease severity in patients with mild to moderate AD. Moreover, the sensitivity to the very mild stages of $\mathrm{AD}$ will be assessed in those $\mathrm{AD}$ patients with highest mini-mental state score ( 26 and above). ${ }^{14}$

\section{METHODS \\ Subjects}

This study comprised 29 patients who met NINCDS-ADRDA criteria for probable $\mathrm{AD}^{15}$ and 26 non-demented controls recruited between September 1993 and December 1994 for a study on linear measures of brain atrophy in $\mathrm{AD}$ and have been described in previous reports. ${ }^{16} \mathrm{AD}$ were outpatients seen at the Alzheimer's Unit, Brescia, Italy. Routine dementia assessment including standardised history, laboratory examinations, physical and neurological examination, neuropsychological assessment, and routine computed tomography was carried out in all. ${ }^{16}$ Control subjects were patients' relatives (mostly spouses) without detectable cognitive deficit. All were given the mini-mental state examination (MMSE) and clinical dementia rating scale ${ }^{17}$ and were judged not to be demented by a neurologist and a psychologist involved in the evaluation of the patients. Uncontrolled physical diseases (heart or renal failure, cirrhosis, etc) were considered exclusion criteria, while risk factors such as hypertension and diabetes were accepted. Of the original 46 patients and 31 controls, ${ }^{16} 17$ and 4 could not be included in this study as some slices were missing. One additional control was excluded for

Abbreviations: $A D$, Alzheimer's disease; MMSE, mini-mental state examination; SPM, statistical parametric mapping; MR, magnetic resonance 
Table 1 Sociodemographic and clinical features of 29 mild to moderate $A D$ patients and 26 non-demented controls

\begin{tabular}{lcc}
\hline & $\begin{array}{c}A D \\
(n=29)\end{array}$ & $\begin{array}{l}\text { Controls } \\
(n=26)\end{array}$ \\
\hline $\begin{array}{l}\text { Age (y) } \\
\text { mean (SD) } \\
\text { range }\end{array}$ & $74(9)$ & $69(8)$ \\
Women $n(\%)$ & $23-86$ & $54-86$ \\
Education (y) & $79)$ & $17(65)$ \\
mean (SD) & $7(4)$ & $8(3)$ \\
range & $2-18$ & $5-19$ \\
$\begin{array}{l}\text { Mini Mental State Exam } \\
\text { mean (SD) }\end{array}$ & $21(4)$ & $29(1)$ \\
range & $12-27$ & $25-30$ \\
Clinical dementia rating scale $n(\%)$ \\
0 \\
0.5 & $8(27.6)$ & $26(100)$ \\
1 & $13(44.8)$ & \\
$2+$ & $8(27.6)$ & \\
\hline &
\end{tabular}

the development of multiple system atrophy a few years after scanning.

Table 1 shows that patients were about five years older $(p=0.04)$ than controls, while sex and education were not dif- ferent $(p>0.09)$. Dementia severity was of mild to moderate degree. All patients and controls were right handed.

Three AD patients were in the highest decile of the MMSE distribution and were selected to investigate atrophy in very mild AD. Age, sex, and MMSE were: 66, F, 26; 81, F, 27; and 79, M, 27.

Written informed consent was obtained by both patients and control subjects or their primary caregivers after discussion of risks and benefits of participation. No compensation was provided. The study was approved by the local ethics committee.

\section{MR images acquisition}

For each subject, a high resolution sagittal Tl weighted volumetric MR scan was acquired at the Radiology Department, University of Verona, using a 1.5 tesla Magnetom scanner (Siemens, Erlangen, Germany), with a gradient echo 3D technique: $\mathrm{TR}=10 \mathrm{~ms}, \mathrm{TE}=4 \mathrm{~ms}, \mathrm{TI}=300 \mathrm{~ms}$, flip angle $=10^{\circ}$, field of view $=250 \mathrm{~mm}$, acquisition matrix $160 \times 256$, slice thickness $1.3 \mathrm{~mm}, 128$ slices. The same parameters were used for every subject.

\section{Image preprocessing}

After removing the voxels below the cerebellum with MRIcro (www.psychology.nottingham.ac.uk/staff/crl/mricro.html), ${ }^{18}$ MR scans were analysed with SPM99 (www.fil.ion.ucl.ac.uk/ spm) running under Matlab 6.0 (Mathworks, Sherborn, MA, USA) on a Sun Sparc Ultra 30 workstation (Sun Microsystem, Mountain View, CA). Before carrying out group comparisons,

Table 2 Regional grey matter density reduction in 29 mild to moderate AD patients compared with 26 non-demented controls

\begin{tabular}{|c|c|c|c|c|c|}
\hline \multirow{2}{*}{$\begin{array}{l}\text { Cluster size k } \\
\left(\mathrm{mm}^{3}\right)\end{array}$} & \multirow[b]{2}{*}{ Region } & \multicolumn{3}{|c|}{ Stereotactic coordinates ( $\mathrm{mm})$} & \multirow[b]{2}{*}{ Z score } \\
\hline & & $x$ & $y$ & z & \\
\hline \multirow[t]{2}{*}{138 (1 104) } & R hippocampus (head)/ amygdala & 32 & -4 & -20 & 5.99 \\
\hline & R hippocampus (head)/ amygdala & 26 & -6 & -14 & 5.79 \\
\hline \multirow[t]{2}{*}{$45(360)$} & $R$ uncus & 20 & 6 & -22 & 5.92 \\
\hline & $\mathrm{R}$ anterior amygdala & 26 & 10 & -26 & 5.84 \\
\hline $45(360)$ & R hippocampus (tail) & 24 & -34 & 4 & 5.60 \\
\hline 43 (344) & $\mathrm{R}$ anterior cingulate & 8 & 34 & 18 & 5.58 \\
\hline $12(96)$ & $\mathrm{L}$ middle temporal gyrus & -56 & -14 & -14 & 5.26 \\
\hline 56 (448) & L hippocampus (head) & -26 & -8 & -16 & 5.21 \\
\hline $13(104)$ & L hippocampus (body) & -36 & -28 & -12 & 5.20 \\
\hline $5(40)$ & L caudate (head) & -10 & 20 & 2 & 5.14 \\
\hline 8 (64) & $\mathrm{R}$ superior temporal gyrus & 54 & -42 & 14 & 5.09 \\
\hline \multirow[t]{2}{*}{$33(264)$} & $\mathrm{R}$ middle temporal gyrus & 52 & -30 & -2 & 5.07 \\
\hline & $\mathrm{R}$ middle temporal gyrus & 54 & -22 & -10 & 4.84 \\
\hline $4(32)$ & L precuneus & -10 & -62 & 34 & 5.03 \\
\hline 9 (72) & R precuneus & 18 & -66 & 32 & 4.96 \\
\hline $4(32)$ & $\mathrm{L}$ middle frontal gyrus & -26 & -10 & 56 & 4.95 \\
\hline $4(32)$ & $\mathrm{L}$ middle frontal gyrus & -28 & 44 & -12 & 4.94 \\
\hline $8(64)$ & R fusiform gyrus & 52 & -40 & -22 & 4.89 \\
\hline $3(24)$ & L precuneus & -10 & -54 & 36 & 4.82 \\
\hline $1(8)$ & R hippocampus (body) & 38 & -24 & -14 & 4.80 \\
\hline $1(8)$ & L insula & -44 & 10 & -2 & 4.78 \\
\hline $1(8)$ & L anterior amygdala & -24 & 8 & -24 & 4.78 \\
\hline $3(24)$ & $R$ uncus & 28 & -8 & -38 & 4.76 \\
\hline $4(32)$ & $\mathrm{R}$ middle temporal gyrus & 52 & -8 & -16 & 4.75 \\
\hline $2(16)$ & $\mathrm{R}$ insula & 44 & -26 & 18 & 4.75 \\
\hline $4(32)$ & R precuneus & 4 & -52 & 30 & 4.75 \\
\hline $1(8)$ & R fusiform gyrus & 58 & -36 & -26 & 4.75 \\
\hline $2(16)$ & $\mathrm{R}$ insula & 50 & -4 & 8 & 4.74 \\
\hline $1(8)$ & R hippocampus (tail) & 38 & -30 & -12 & 4.74 \\
\hline $1(8)$ & L posterior cingulate & 0 & -46 & 44 & 4.71 \\
\hline 1 (8) & $\mathrm{R}$ insula & 38 & 4 & 12 & 4.71 \\
\hline $1(8)$ & R hippocampus (tail) & 36 & -32 & -10 & 4.70 \\
\hline $1(8)$ & R superior temporal gyrus & 54 & -58 & 28 & 4.69 \\
\hline 1 (8) & L precuneus & -30 & -70 & 34 & 4.69 \\
\hline
\end{tabular}

$\mathrm{L}=$ left, $\mathrm{R}=$ right. The first line denotes the presence of a $3 \mathrm{D}$ cluster made of 138 contiguous voxels of significantly decreased ( $p<0.05$ corrected for multiple comparisons) grey matter density. The most significant voxel of the cluster has stereotactic coordinates of $32,-4$, and -20 and is located in the region of the right hippocampal/amygdalar complex. Within the same cluster there is a second peak of significance distant more than $8 \mathrm{~mm}$ from the former and located at $26,-6,-14$. 


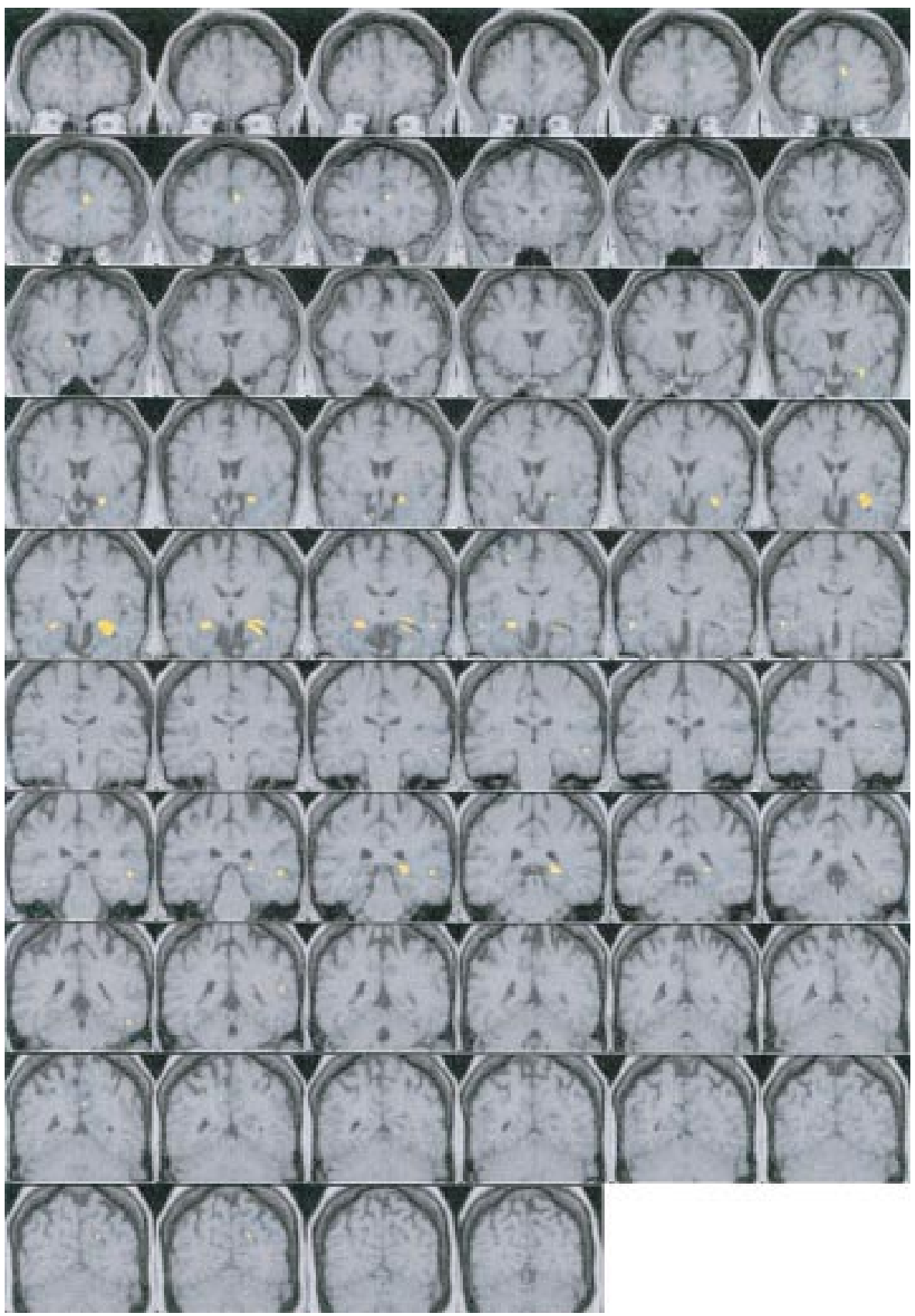

Figure 1 Voxels of decreased grey matter density in the 29 mild to moderate AD patients compared with the 26 non-demented controls. The first and last slices are those where the anteriormost and posteriormost significant voxels are detected. Significant voxels are superimposed on MR images of a single subject. Right on image is right in the brain.

three steps need to be carried out with SPM99: creation of a grey matter template, normalisation of the grey matter to the template, and smoothing. A complete description of the preprocessing procedures can be found elsewhere. ${ }^{6}$

\section{Grey matter template}

This was made through creation of a customised template of the whole brain, normalisation of the original images to the template, extraction of the grey matter, smoothing with a 12 mm filter, and averaging. The customised template of the whole brain was obtained through normalisation of the images of all patients and controls to the Tl stereotactic template of SPM99 $^{19}$ using a 12 parameter affine transformation followed by smoothing with an $8 \mathrm{~mm}$ isotropic Gaussian kernel and averaging of the smoothed normalised images. Starting estimates for the registration were assigned by specifying the position of the anterior commissure. $^{20}$

The normalisation of the original images to the template was obtained through a 12 parameter affine and non-linear transformation. Grey matter was then extracted through segmentation of images into grey matter, white matter, and cerebrospinal fluid with a modified mixture model cluster algorithm, smoothed with a $12 \mathrm{~mm}$ filter, and averaged thus obtaining a stereotactic customised grey matter template. Customised priors were computed from this template that will be used to improve the accuracy of the extraction of the grey matter of each participant in the following normalisation step. $^{13}$ 
Table 3 Regional grey matter density reduction in 3 very mild AD patients (MMSE of 26 and 27) compared with 26 non-demented controls $(p<0.0001$ uncorrected)

\begin{tabular}{llllll}
\hline \multirow{2}{*}{$\begin{array}{l}\text { Cluster size } \mathrm{k} \\
\left(\mathrm{mm}^{3}\right)\end{array}$} & Region & \multicolumn{3}{l}{ Stereotactic coordinates $(\mathrm{mm})$} & \\
\cline { 3 - 5 } & $\mathrm{x}$ & $\mathrm{y}$ & $\mathrm{z}$ & Z score \\
\hline $29(232)$ & R hippocampus (head)/ amygdala & 24 & -4 & -22 & 4.19 \\
$5(40)$ & L middle frontal gyrus & -24 & 44 & -12 & 4.10 \\
$13(104)$ & L hippocampus (head)/ amygdala & -28 & -2 & -22 & 4.02 \\
$6(48)$ & L middle temporal gyrus & -34 & 10 & -40 & 4.02 \\
$1(8)$ & R inferior temporal gyrus & 58 & -54 & -12 & 3.87 \\
$5(40)$ & L middle temporal gyrus & -32 & 0 & -42 & 3.83 \\
$1(8)$ & R precentral gyrus & 62 & 4 & 26 & 3.81 \\
$2(16)$ & L medial frontal gyrus & -8 & 38 & -8 & 3.74 \\
\hline L=left, R=right. & & & & & \\
\hline
\end{tabular}

\section{Normalisation}

The grey matter of patients was extracted with the same procedure as described above for controls. The grey matter of patients and controls was normalised onto the grey matter template with affine and non-linear transformations, medium regularisation, reslicing $2 \times 2 \times 2 \mathrm{~mm}$, and no masking. ${ }^{10}$ The resulting images were visually inspected one by one to exclude macroscopical segmentation errors.

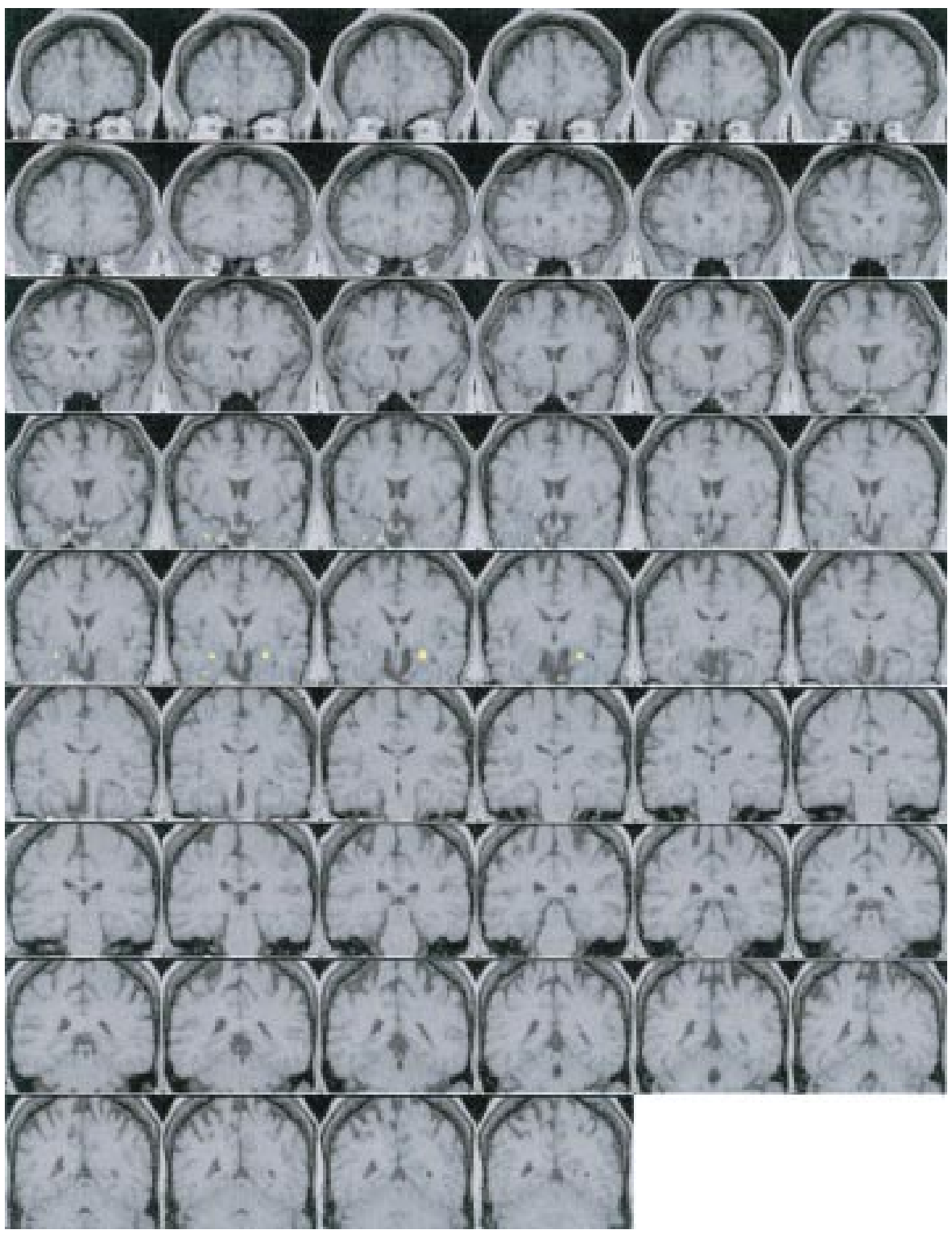

Figure 2 Voxels of decreased grey matter density in the three very mild AD patients (MMSE of 26 and 27) compared with the 26 non-demented controls. The first and last slices are those where the anteriormost and posteriormost significant voxels are detected. Significant voxels are superimposed on MR images of a single subject. Right on image is right in the brain. 
Table 4 Correlation of disease severity (MMSE scores) with grey matter density in the 29 AD patients $(p<0.0001$ uncorrected)

\begin{tabular}{llllll}
\hline \multirow{2}{*}{$\begin{array}{l}\text { Cluster size } \mathrm{k} \\
\left(\mathrm{mm}^{3}\right)\end{array}$} & Region & \multicolumn{3}{l}{ Stereotactic coordinates $(\mathrm{mm})$} & \\
\cline { 3 - 5 } & $\mathrm{x}$ & 60 & -16 & -32 & $\mathrm{z}$ \\
\hline $14(112)$ & R inferior temporal gyrus & 52 & -50 & 20 & 4.05 \\
$8(64)$ & R superior temporal gyrus & 60 & -50 & 10 & 3.97 \\
$5(40)$ & R superior temporal gyrus & -2 & -16 & 40 & 3.93 \\
$7(56)$ & L posterior cingulate & 24 & -64 & 46 & 3.89 \\
$1(8)$ & R superior parietal lobule & -4 & -74 & 34 & 3.80 \\
$1(8)$ & L precuneus & -2 & -38 & 46 & 3.75 \\
$1(8)$ & L precuneus & & & & \\
\hline L=left, R=right. & & & &
\end{tabular}

\section{Smoothing}

The normalised grey matter images were smoothed with an isotropic Gaussian filter with a full width at half maximum of $8 \mathrm{~mm}$.

\section{Statistical analysis}

Two statistical models were built to compare grey matter density between all patients and controls and between the three very mild patients and controls with the "Compare populations-AnCova" procedure. A separate model was built to detect the regions where grey matter density was associated with dementia severity with the "Single subject-Covariates only" procedure. Proportional scaling was not used and grey matter thresholding was set at 0.8 .

In the first two models the contrast of interest was that of decreased grey matter density of patients compared with controls and age was entered as a nuisance covariate. In the first model (all patients versus controls) the significance threshold of the $t$ statistics was set at 0.05 corrected for multiple comparisons. In the second model (the three very mild patients versus controls) the significance threshold was set at a higher level (0.0001 uncorrected) to compensate for the small sample size. The anatomical localisation of the $t$ statistics peaks was made based on visual examination with the aid of a brain atlas. ${ }^{21}$ In the third model, MMSE was entered as the covariate of interest. The significance threshold of the $t$ statistics was set at 0.0001 uncorrected.

\section{RESULTS}

Table 2 and figure 1 show the results of the comparison between all patients and controls. The table shows that the largest regions of significant grey matter density reduction were located bilaterally in the medial temporal lobe structures (hippocampus, amygdala, and uncus). Grey matter density reduction was significant in all parts of the hippocampus (head, body, and tail). Figure 1 shows that the largest cluster located in the head of the right hippocampus and amygdala tended to involve the two structures separately. Other smaller areas of grey matter density reduction were located in the temporal lobe (superior and middle temporal gyri and fusiform gyrus), parietal lobe (precuneus), mesial aspect of the brain (anterior and posterior cingulate), and insular cortex. Scattered but significant voxels were detected in the head of the caudate nucleus and frontal gyri. Overall, the structures on the right side were more heavily involved than those on the left.

The results were checked in a subgroup of 17 patients and 17 controls individually matched ( \pm 2 years) for age (mean (SD) ages 72.5 (8.7) and 73.0 (8.4) respectively). The largest and most significant clusters were confirmed in the medial temporal lobe (left hippocampal head/posterior amygdala at $-26,-8,-14$ with $\mathrm{z}=5.07$ and right anterior amygdala at 22,4 , -20 with $\mathrm{z}=4.96$ ).
Table 3 and figure 2 show the results of the comparison between the three patients with the highest MMSE and controls. Medial temporal regions on both sides were again the most significant or largest regions. Smaller regions of grey matter density reduction were detected in the temporal and frontal gyri. One voxel resulted significant in the precentral gyrus. To detect false positive voxels that might arise from the comparatively low significance threshold, we checked which voxels were negative in the first experiment, where a much stricter $p$ threshold was used $(p=0.05$ corrected). We found that the only surviving voxels were located in the hippocampal/amygdalar region.

The results were checked with an age matched group of 14 controls with age range ( 66 to 81 years) equal to that of the three very mild $\mathrm{AD}$ patients (mean (SD) ages 74.2 (5.5) and 75.3 (6.7) respectively). The largest and most significant cluster was detected in the left amygdala (at $-30,4,-24$ with $\mathrm{z}=4.89$ ).

The areas where grey matter density reduction was correlated with dementia severity (table 4 and fig 3 ) corresponded to the temporoparietal cortex mainly to the right involving the superior and inferior temporal gyri, parietal lobule, and precuneus. A comparatively large region was also detected in the left posterior cingulate. The grey matter density values of the most significant voxels in the right and left regions showed a significant correlation with MMSE scores (fig 4).

All the analyses were re-run by covarying by sex, but the results did not change appreciably.

\section{DISCUSSION}

We have shown that voxel based morphometry with SPM: (1) is sensitive to medial temporal grey matter density reduction in $\mathrm{AD}$ and that this is the case even in the very mild patients; (2) demonstrates grey matter density reduction outside the medial temporal lobe confined to areas in the temporal gyri, precuneus, insular and cingulate cortex, and caudate nucleus; (3) is sensitive to the temporoparietal atrophy associated with the progression of the disease. These results support the use of this tool to study regional atrophy in AD.

Three studies have performed voxel based morphometry in AD. In seven comparatively young AD patients (mean age of 65 years) with mild to moderate $\mathrm{AD}$ compared with seven controls, Rombouts et al ${ }^{11}$ found atrophy of the hippocampus, insula, and, unexpectedly, caudate nucleus. In the hippocampus and insula, atrophy tended to be greater on the right. The authors used a moderately restrictive criterion for atrophy detection ( $\mathrm{p}<0.0005$ uncorrected for multiple comparisons). Baron et $a l^{10}$ have studied 19 mild to moderate $\mathrm{AD}$ patients and compared them with 16 controls finding atrophy in the medial temporal structures and cingulate, insular, and temporoparietal cortex. Medial temporal atrophy was centred in the amygdalae on both sides although more marked on the right, 


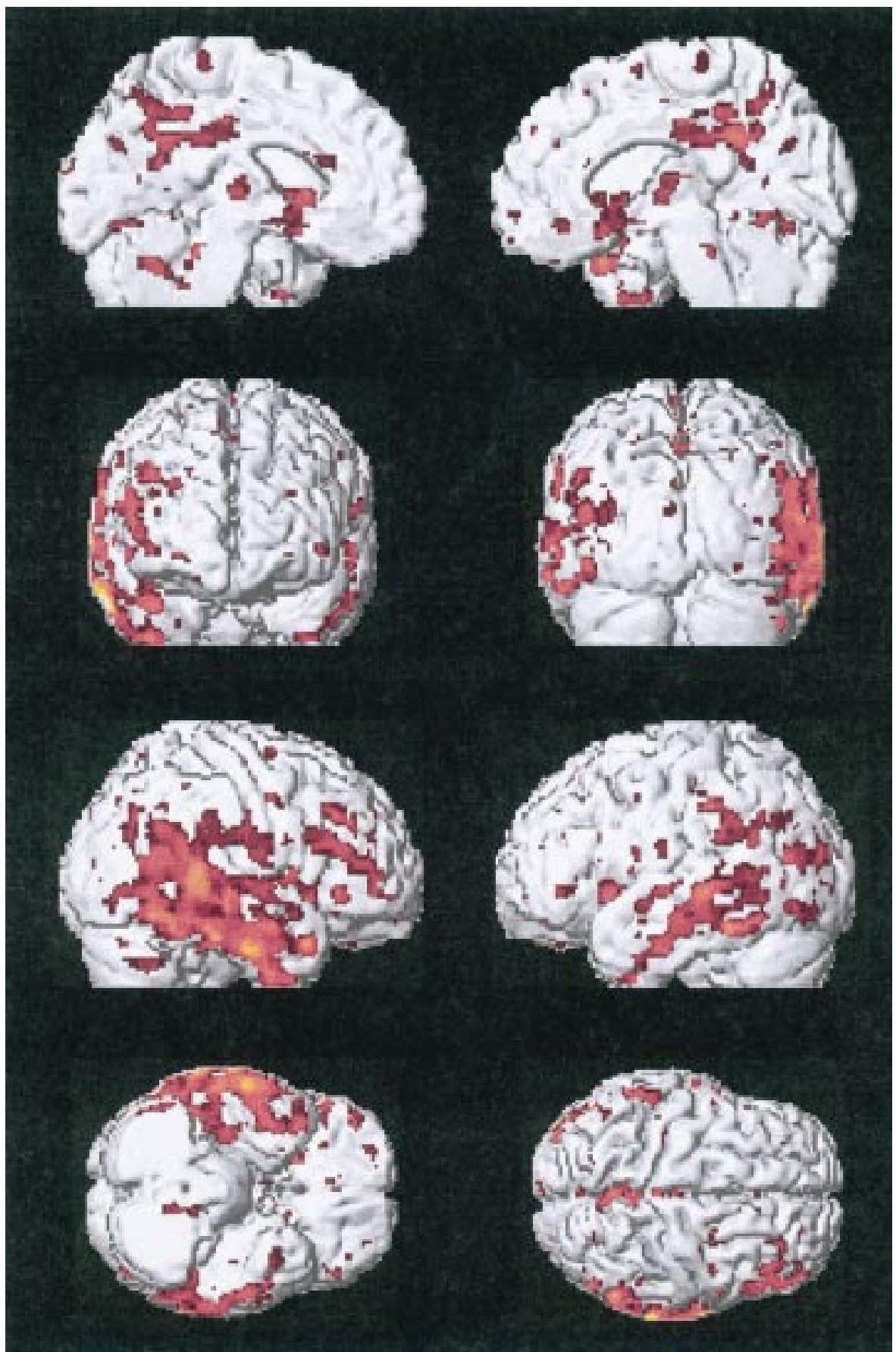

Figure 3 Correlation of disease severity (MMSE scores) with grey matter density in the 29 AD patients. Significant voxels (threshold at $p<$ 0.05 uncorrected for illustrative purposes only) are projected on the surface rendered brain of a single subject.

where also the posterior hippocampus was involved. The criterion for atrophy detection was $\mathrm{p}<0.10$ corrected for multiple comparisons. Ohnishi et al ${ }^{12}$ compared 26 mild with moderate $\mathrm{AD}$ patients to 92 controls and found atrophy remarkably confined to the whole length of the hippocampus bilaterally but more marked on the right side. The SPM parameters used to obtain these results are not described in sufficient detail to permit comparisons with other works. All the studies have used as template brain the one computed at the Montreal Neurological Institute resulting from averaging non-atrophic brains of 152 young adults (mean age 25 years). ${ }^{19}$

In this study, we have assessed a patient group representative of the population of sporadic $\mathrm{AD}$ (mean age 74 years) and used a preprocessing protocol (customised template and customised priors computed from our study groups) that should optimise the normalisation and segmentation of atrophic brains. We have confirmed all of the previous findings (atrophy of head body and tail of the hippocampus, amygdala, insula, lateral temporal and cingulate cortex, precuneus, and caudate nucleus) and have found evidence of involvement of other regions demonstrated to be affected in pathological and structural studies of AD patients of mild to moderate severity (uncus and fusiform gyrus). ${ }^{22} 23$

The finding of atrophy of the anterior cingulate gyrus was unexpected as most studies have found involvement of the posterior cingulate gyrus. ${ }^{24}{ }^{25}$ However, the anterior cingulate gyrus is involved in complex attentional tasks, ${ }^{26}$ and attention is known to be the first non-memory domain to be affected in $\mathrm{AD}$, before deficits in language and visuospatial functions appear. ${ }^{27}$ With the use of structural and functional imaging, 

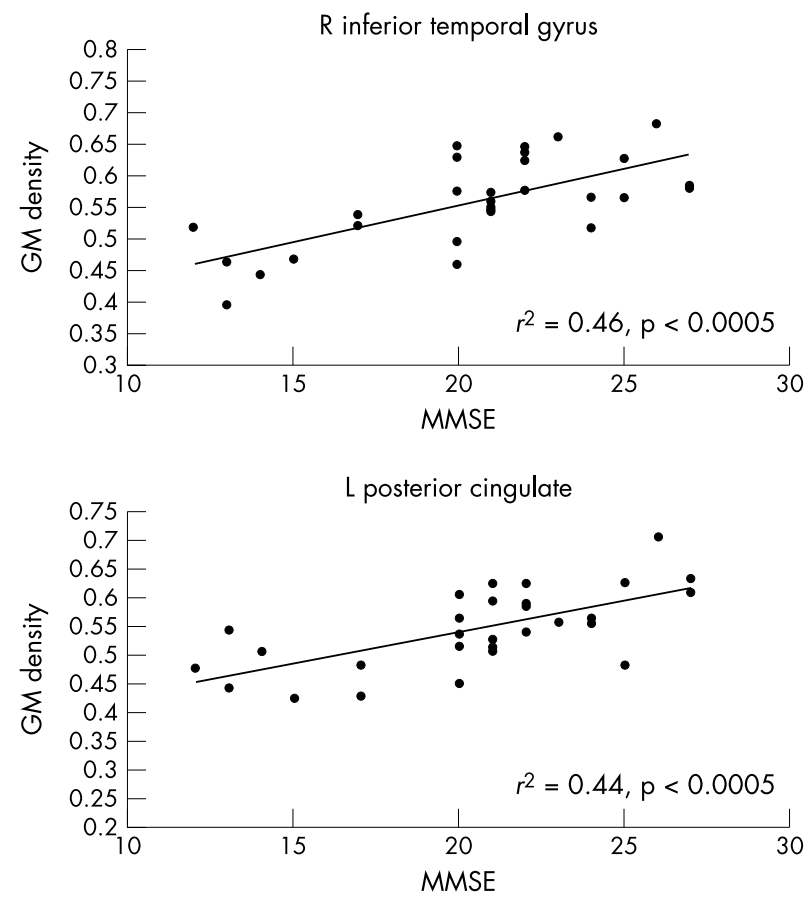

Figure 4 Correlation of dementia severity (MMSE score) with grey matter density in right (stereotactic coordinates $60,-16,-32$ ) and left regions (stereotactic coordinates $-2,-16,40$ ) in the $29 A D$ patients.

other investigators have found that anterior cingulate atrophy is predictive of conversion to $\mathrm{AD}$ in memory impaired individuals, ${ }^{28} 29$ suggesting that this region might be affected relatively early in the course of the disease.

Caudate atrophy in AD has been reported by other investigators with voxel based morphometry ${ }^{10}$ and has been explained in view of the demonstrated involvement of striatial nuclei by neurofibrillary changes. ${ }^{30}$ Recently, in 21 AD patients with a mean MMSE of 16, Barber and colleagues have found a $9 \%-14 \%$ reduction of the volume of the caudate nucleus. ${ }^{31}$ Further evidence that the caudate nucleus is abnormal in $\mathrm{AD}$ comes from MR studies showing an increase of iron lad comparable to that of Huntington's disease. ${ }^{32}$

The findings in the subgroup of three mildest AD patients are largely but not completely overlapping with those in the whole group. The comparatively looser significance threshold that we used $(\mathrm{p}<0.0001$ uncorrected versus $\mathrm{p}<0.05$ corrected), might lead to the hypothesis that some significant voxels might be false positives. However, with the exception of one voxel in the right precentral gyrus and two in the left medial frontal gyrus, all other positive voxels were located in regions found atrophic also in the experiment with the larger group. Moreover, the voxels in the right precentral and left medial frontal gyrus were those with the lowest significance values (table 3). Lastly, when a voxel by voxel comparison was performed between the results of the two analyses, voxels significant in both analyses were located in the medial temporal region. This supports the view that voxel based morphometry with SPM might be sensitive to atrophy even in the mildest phases of AD, although present algorithms are still unsatisfactorily specific. Moreover, these data will need to be confirmed in a study where significant voxels survive correction for multiple comparisons, which will probably require large number of mild AD cases.

Although the regions that we found correlated with global cognitive severity are those expected based on current knowledge, we found a striking and unexpected asymmetry (right greater than left). This is not in agreement with the notion that the MMSE is heavily weighted on verbal rather than non-verbal tasks and with most previous studies with functional ${ }^{33}$ and traditional structural imaging. ${ }^{34}$ However, others have indicated that the MMSE is more affected by right than left focal hemispheric lesions ${ }^{35}$ and that structural markers of severity in $\mathrm{AD}$ are more strictly correlated with right than left temporoparietal hypometabolism. ${ }^{36}$ To our knowledge, this is the first study of the correlation between MMSE and atrophy with voxel based morphometry, and replication is mandatory.

The main limitation of this study relates to the arbitrary setting of the significance threshold for the detection of grey matter density reduction. However, this is an intrinsic limitation of SPM and studies published to date have all used different thresholds. ${ }^{1011237}$ For each voxel SPM computes a $t$ statistics denoting the difference of mean grey matter density between two groups. As is the case of the traditional $t$ statistics, probability is sensitive to group size in that experiments with larger groups have more power to detect effect differences than experiments with smaller groups. In experiments with smaller groups or effect size, the lower power can be accounted for by setting a lower probability threshold. This increases sensitivity, but at the expense of lower specificity. This problem is particularly cogent to the correlation between MMSE and atrophy, where the poor specificity is an unsolved problem. The implementation of Bayesian analysis into SPM will help investigate this issue. ${ }^{38}$

The quest for observer independent tools to assess brain changes in degenerative brain diseases has just begun. The increasing use of voxel based morphometry with SPM in brain disorders ${ }^{39-43}$ is attributable to the high independence from the human operator in that once the preprocessing analysis parameters and the significance threshold of the analysis have been set, the process is largely automatic. However, its advantages and limitations are only beginning to be elucidated and more work will be necessary. The development of other techniques such as deformation based and tensor based ${ }^{4-6}$ as alternatives to or integrated into the voxel based morphometry approach $^{6}$ might provide additional information that will hopefully prove useful in clinical and research settings.

\section{ACKNOWLEDGEMENTS}

We are indebted to Dr Maria Luisa Gorno Tempini for valuable comments on SPM analysis.

\section{Authors' affiliations}

G B Frisoni, C Testa, A Zorzan, F Sabattoli, Laboratory of Epidemiology and Neuroimaging, IRCCS San Giovanni di Dio-FBF, Brescia, Italy

A Beltramello, Service of Neuroradiology, Ospedale Maggiore, Verona, Italy

H Soininen, Department of Neurology, Kuopio University Hospital, Kuopio, Finland

M P Laakso, Department of Neurology and Clinical Radiology, Kuopio University Hospital

Funding: this study was funded by the Research Council for Health of the Academy of Finland, and EVO grants 5510, 5772722 from the Kuopio University Hospital.

Competing interests: none declared.

\section{REFERENCES}

I Jack CR Jr, Petersen RC, Xu YC, et al. Medial temporal atrophy on MRI in normal aging and very mild Alzheimer's disease. Neurology 1997;49:786-94.

2 Bobinski $M$, de Leon $M$, Convit $A$, et al. MRI of entorhinal cortex in mild Alzheimer's disease. Lancet 1999;353:38-40.

3 Jack CR Jr, Petersen RC, Xu YC, et al. Prediction of AD with MRI-based hippocampal volume in mild cognitive impairment. Neurology 1999;52:1397-403

4 Toga AW. Brain warping. Toronto: Academic Press, 1999

5 Csernansky JG, Wang L, Joshi S, et al. Early DAT is distinguished from aging by high-dimensional mapping of the hippocampus. Dementia of the Alzheimer type. Neurology 2000;55:1636-43. 
6 Ashburner J, Friston K J. Voxel-based morphometry-the methods. Neurolmage 2000;11:805-21.

7 Fox NC, Freeborough PA. Brain atrophy progression measured from registered serial MRI: validation and application to Alzheimer's disease. J Magn Reson Imaging 1997;7:1069-75.

8 Frackowiak RSJ, Friston KJ, Frith CD, et al. Human brain function. Toronto: Academic Press, 1997.

9 Friston KJ, Frith CD, Liddle PF, et al. The relationship between global and local changes in PET scans. J Cereb Blood Flow Metab 1990; 10:458-66.

10 Baron JC, Chételat G, Desgranges B, et al. In vivo mapping of gray matter loss with voxel-based morphometry in mild Alzheimer's disease. Neurolmage 2001;14:298-309.

11 Rombouts SA, Barkhof F, Witter MP, Scheltens P. Unbiased whole-brain analysis of gray matter loss in Alzheimer's disease. Neurosci Lett 2000;285:231-3.

12 Ohnishi T, Matsuda H, Tabira T, et al. Changes in brain morphology in Alzheimer disease and normal ageing: is Alzheimer disease an exaggerated aging process? ANNR Am J Neuroradiol 2001;22:1680-5.

13 Good CD, Johnsrude IS, Ashburner J, et al. A voxel-based morphometric study of ageing in 465 normal adult human brains. Neurolmage $2001 ; 14: 21-36$

14 Folstein MF, Folstein SE, McHugh PR. "Mini-mental state". A practical method for grading the cognitive state of patients for the clinician. $J$ Psychiat Res 1975;12:189-98.

15 McKhann G, Drachman D, Folstein MF, et al. Clinical diagnosis of Alzheimer's disease: report of the NINCDS-ADRDA Work Group under the auspices of Department of Health and Human Services Task Force on Alzheimer's Disease. Neurology 1984;34:939-44.

16 Frisoni GB, Beltramello A, Weiss C, et al. Linear measures of atrophy in mild Alzheimer's disease. ANNR Am J Neuroradiol 1996;17:913-23.

17 Hughes CP, Berg L, Danziger WL, et al. A new clinical scale for the staging of dementia. Br J Psychiatry 1982;140:566-72.

18 Rorden C, Brett M. Stereotaxic display of brain lesions. Behav Neurol 2001;112:191-200.

19 Evans AC, Kamber M, Collins DL, et al. An MRI-based probabilistic atlas of neuroanatomy. In: Shorvon S, Fish D, Andermann F, et al, eds. Magnetic resonance scanning and epilepsy. New York: Plenum Press 1994:263-74.

20 Ashburner J, Neelin P, Collins DL, et al. Incorporating prior knowledge into image registration. Neurolmage 1997;6:344-52.

21 Mai JK, Asshever J, Paxinos G. Atlas of the human brain. New York: Academic Press, 1997.

22 Esiri MM, Pearson RC, Steele JE, et al. A quantitative study of the neurofibrillary tangles and the choline acetyltransferase activity in the cerebral cortex and the amygdala in Alzheimer's disease. J Neurol Neurosurg Psychiatry 1990;53:161-5.

23 Convit A, de Asis J, de Leon M, et al. Atrophy of the medial occipitotemporal, inferior, and middle temporal gyri in non-demented elderly predict decline to Alzheimer's disease. Neurobiol Aging 2000;21:19-26.

24 Minoshima S, Giordani B, Berent S, et al. Metabolic reduction in the posterior cingulate cortex in very early Alzheimer's disease. Ann Neurol 1997;42:85-94.
25 Vogt BA, Van Hoesen GW, Vogt LJ. Laminar distribution of neuron degeneration in posterior cingulate cortex in Alzheimer's disease. Acto Neuropathol (Berl) 1990;80:581-9.

26 Mesulam MM, Nobre AC, Kim YH, et al. Heterogeneity of cingulate contributions to spatial attention. Neurolmage 200 1;13:1065-72.

27 Matsuda H. Cerebral blood flow and metabolic abnormalities in Alzheimer's disease. Ann Nucl Med 2001;15:85-92.

28 Killiany RJ, Gomez-lsla T, Moss M, et al. Use of structural magnetic resonance imaging to predict who will get Alzheimer's disease. Ann Neurol 2000;47:430-9

29 Johnson KA, Jones K, Holman BL, et al. Preclinical prediction of Alzheimer's disease using SPECT. Neurology 1998;50:1563-71.

30 Braak H, Braak E. Alzheimer's disease: striatal amyloid deposits and neurofibrillary changes. J Neuropathol Exp Neurol 1990;49:215-24.

31 Barber R, McKeith I, Ballard C, et al. Volumetric MRI study of the caudate nucleus in patients with dementia with Lewy bodies, Alzheimer's disease, and vascular dementia. J Neurol Neurosurg Psychiatry 2002;72:406-7.

32 Bartzokis G, Tishler TA. MRI evaluation of basal ganglia ferritin iron and neurotoxicity in Alzheimer's and Huntingon's disease. Cell Mol Biol 2000;46:82 1-33.

33 Imran MB, Kawashima R, Awata S, et al. Tc-99m HMPAO SPECT in the evaluation of Alzheimer's disease: correlation between neuropsychiatric evaluation and CBF images. J Neurol Neurosurg Psychiatry 1999:66:228-32.

34 Smith CD, Malcein M, Meurer K, et al. MRI temporal lobe volume measures and neuropsychologic function in Alzheimer's disease. $J$ measures and neuropsychologic

35 Grace J, Nadler JD, White DA, et al. Folstein vs modified Mini-Mental State Examination in geriatric stroke. Stability, validity, and screening utility. Arch Neurol 1995;52:477-84.

36 Meguro K, LeMestric C, Landeau B, et al. Relations between hypometabolism in the posterior association neocortex and hippocampal atrophy in Alzheimer's disease: a PET/MRI correlative study. J Neurol Neurosurg Psychiatry 2001;71:315-21

37 Van Laere KJ, Dierckx RA. Brain perfusion SPECT: age- and sex-related effects correlated with voxel-based morphometric findings in healthy adults. Radiology 2001;221:810-17.

38 Friston KJ, Penny W, Phillips C, et al. Classical and Bayesian Inference in Neuroimaging: Theory. Neurolmage 2002;16:465-83.

39 Thieben MJ, Duggins AJ, Good CD, et al. The distribution of structural neuropathology in pre-clinical Huntington's disease. Brain 2002;125:1815-28.

40 Kaufmann C, Schuld A, Pollmacher T, et al. Reduced cortical gray matter in narcolepsy: preliminary findings with voxel-based morphometry. Neurology 2002;58: 1852-5.

41 Kassubek J, Juengling FD, Hellwig B, et al. Thalamic gray matter changes in unilateral Parkinsonian resting tremor: a voxel-based morphometric analysis of 3-dimensional magnetic resonance imaging. Neurosci Lett 2002;323:29-32.

42 Franklin TR, Acton PD, Maldjian JA, et al. Decreased gray matter concentration in the insular, orbitofrontal, cingulate, and temporal concentration in the insular, orbitofrontal, cingulate, and temporal
cortices of cocaine patients. Biol Psychiatry 2002;51:134-42.

43 Rosen HJ, Kramer JH, Gorno-Tempini ML, et al. Patterns of cerebral atrophy in primary progressive aphasia. Am J Geriatr Psychiatry 2002; 10:89-97. 